\title{
Protective effects of benfotiamine on irisin activity in methotrexate-induced liver injury in rats
}

Mehmet Ali Erdogan ${ }^{1}$, Alper Yalcin²

\author{
${ }^{1}$ Department of Gastroenterology, Faculty of Medicine, Inonu University, Malatya, \\ Turkey \\ 2Department of Histology and Embryology, Faculty of Medicine, Adiyaman University, \\ Adiyaman, Turkey
}

Submitted: 20 September 2018

Accepted: 27 October 2018

Arch Med Sci 2020; 16 (1): 205-211

DOI: https://doi.org/10.5114/aoms.2018.80002

Copyright (c) 2018 Termedia \& Banach

\section{Abstract}

Introduction: Methotrexate (MTX) causes hepatotoxicity by producing oxidative stress. Benfotiamine and irisin have protective effects against oxidative stress. The aim of this study was to investigate the changes in irisin activity in the liver as a result of toxicity produced by MTX and the protective role of benfotiamine in the hepatotoxicity.

Material and methods: Rats were divided into 4 groups as follows: control, benfotiamine (50 mg/kg, oral gavage (o.g.), for 14 days), MTX (MTX $20 \mathrm{mg} / \mathrm{kg}$ intraperitoneally (i.p.) on day 1), MTX + benfotiamine (MTX $20 \mathrm{mg} / \mathrm{kg}$ (i.p.) on day 1 , then $50 \mathrm{mg} / \mathrm{kg}$ (o.g.) benfotiamine for 14 days). Liver tissue was used to examine histopathological and immunohistochemical changes. Serum was used to look for oxidative stress markers (total antioxidant status (TAS) and total oxidant status (TOS)).

Results: Administration of MTX caused a significant TOS increase and TAS decrease in the serum as compared to the control group. Immunohistochemically, irisin was significantly increased in immunoreactivity in the MTX group as compared to the control group $(p<0.05)$. Significant histopathological improvement and decrease in serum TOS levels were observed in the MTX + benfotiamine group compared to the MTX group $(p<0.05)$. In addition, an increase in TAS level and a decrease in irisin immunoreactivity were observed but they were not statistically significant $(p>0.05)$.

Conclusions: Our results showed that MTX caused an increase in the activity of irisin after producing toxicity in the liver. In addition, we found that benfotiamine was effective in preventing damage caused by MTX in the liver.

Key words: irisin, liver toxicity, benfotiamine, oxidative stress, rats.

\section{Introduction}

Methotrexate (MTX) has been used in the treatment of autoimmune diseases such as psoriasis and rheumatoid arthritis, especially leukaemia and various solid tumours, for many years. Even though the mechanism of liver damage caused by MTX is not yet clear, many parameters such as genetic, molecular, and apoptotic factors are thought to play a role in the pathogenesis of hepatotoxicity. Methotrexate is an antimetabolite and folate antagonist. This function is performed by binding to dihydrofolate reductase, which plays a key role in cell replication, and inhibiting the synthesis of tetrahydrofolate required for the formation of purines and

\author{
Corresponding author: \\ Mehmet Ali Erdogan \\ Department \\ of Gastroenterology \\ Inonu University \\ Medical Faculty \\ 44210 Malatya, Turkey \\ Phone: +90 5054965191 \\ Fax: 08502979003 \\ E-mail: mehmet_ali \\ erdogan@hotmail.com
}


pyrimidines. Inhibition of purine and pyrimidine synthesis leads to DNA damage resulting in apoptosis [1, 2]. In addition, MTX causes weakening of the effectiveness of the antioxidant defence system, which protects cells against oxidative stress [3]. Furthermore, MTX also shows a negative effect on energy metabolism by decreasing ATP synthesis [4].

Irisin, which plays a role in energy regulation, is a proteolytic product of fibronectin type III domain 5 (FNCD5), which is a membrane protein in the muscles and a member of the myokine group. After synthesis of irisin, having thermogenic properties, the white fat tissue turns into a brown fat tissue which results in energy dissipation $[5,6]$. The increment in expression of PGC1, also known as the coactivator of peroxisome proliferator-activated receptor (PPAR), leads to an increase in FNDC5, which leads to upregulation of UCP-1 from the brown adipose tissue. It has also been demonstrated that $\mathrm{PGCl}$ stimulates mitochondrial biogenesis, which is known to have beneficial effects on inflammation and many chronic diseases [6].

A fat soluble form of thiamine pyrophosphate, which plays an important role in energy metabolism, is called benfotiamine [7, 8]. Having anti-inflammatory properties, benfotiamine prevents apoptosis caused by high glucose $[9,10]$. It has also been shown in recent studies that it has an antioxidant effect against oxidative stress [11, 12].

Oxidative stress is caused by deterioration of the balance between reactive oxygen species (ROS) and antioxidant defence systems such as superoxide dismutase, glutathione peroxidase and cytochrome oxidase, in favour of ROS. The excess ROS increase causes a toxic effect in the cell and affects the cell components: DNA, protein and lipids [13, 14]. It has been shown in recent studies that oxidative stress increases in relation to age and is responsible for the pathogenesis of many diseases such as atherosclerosis, thyroid diseases, cardiovascular diseases and liver diseases [13, 15-17].

In this study, we aimed to investigate the activity of irisin in liver toxicity due to MTX and the effect of benfotiamine in preventing the damage.

\section{Material and methods}

\section{Animals}

The present study was approved by the Institutional Ethics Committee for Animal Experiments (2018/007). In our study, 28 Wistar Albino male rats weighing 200-250 g and 10-12 weeks old were used by dividing them into 4 groups, each group having 7 animals. The rats were kept in rooms with $22 \pm 2^{\circ} \mathrm{C}$ room temperature, with a $12 \mathrm{~h}$ light and $12 \mathrm{~h}$ dark light cycle, and were provided with feed and water ad libitum. The weights of the animals were determined at the beginning and end of the study, which was to be continued for 14 days in total. The rats in the study were divided into four groups as follows.

1. Group I (control group) $(n=7)$ : no procedure was performed during the experiment.

2. Group II (MTX group) $(n=7)$ : only one dose of $20 \mathrm{mg} / \mathrm{kg}$ MTX (i.p.) was administered on day 1 of the experiment.

3. Group III (benfotiamine group) $(n=7): 50 \mathrm{mg} /$ $\mathrm{kg}$ benfotiamine was given by oral gavage daily for 14 days of the experimental period.

4. Group IV (MTX + benfotiamine group) $(n=7)$ : rats that were given a single dose of $20 \mathrm{mg}$ / $\mathrm{kg}$ (i.p.) MTX on the first day of the experiment were then administered a dose of $50 \mathrm{mg} / \mathrm{kg}$ benfotiamine by oral gavage daily for 14 days of the experimental period. At the end of the experimental period, all rats were decapitated. Then, serum and tissue samples were obtained.

\section{Biochemical study - total antioxidant status (TAS) and total oxidant status (TOS) measurements}

The levels of serum TAS were measured using the Rel Assay Total Antioxidant Status Test Kit in the Olympus AU2700 Autoanalyzer (Mega Medicine San. Ve Tic. Ltd. Sti., Gaziantep, Turkey) [18].

Working Principle: The antioxidants in the example change the concentrated dark blue-green ABTS+ $(30 \mathrm{mmol} / \mathrm{l}) \quad\left[2,2^{\prime}\right.$-azinobis-(3-ethylbenzothiazoline-6-sulfonic acid)] in acetate buffer (0.4 mol/l, pH: 3.6$)$ solution to a reduced colourless ABTS form. The ABTS molecule reduces and decolorizes when diluted with a solution of acetate buffer ( $0.4 \mathrm{~mol} / \mathrm{l}, \mathrm{pH}$ 5.8) having high $\mathrm{pH}$, due to antioxidants present in the samples. There is an inverse relationship between concentration and decolourization of the antioxidant substances found in the samples. The absorbance change detected spectrophotometrically is related to the level of antioxidant in the sample. Stable antioxidant is calibrated with its standard solution (Trolox equivalent). The reaction rate is adjusted with Trolox, which is a vitamin E analogue, and the unit is mmol Trolox equivalent/l [18].

The levels of serum TAS were measured using the Rel Assay Total Antioxidant Status Test Kit in the Olympus AU2700 Autoanalyzer (Mega Medicine San. Ve Tic. Ltd. Sti., Gaziantep, Turkey) [19].

Working principle: oxidizing agents present in the sample oxidize the ferrous ion chelator complex to ferric ion. The reaction is carried out by the oxidizing molecules. The ferric ion forms a coloured complex with the chromogen present in the acidic medium. Density of the colour measured spectrophotometrically is directly proportional to the amount of oxidizing substances present in the 
samples. This measurement method is calibrated with hydrogen peroxide and the results are measured in micromolar hydrogen peroxide equivalent per litre ( $\mu \mathrm{mol} \mathrm{H}_{2} \mathrm{O}_{2}$ equiv./l).

\section{Immunohistochemistry}

The sections cut from paraffin blocks in 4$6 \mu \mathrm{m}$ thickness were placed on polylysine coated slides. The deparaffinized tissues were passed through graded alcohol series and boiled in a microwave oven $(750 \mathrm{~W})$ for $7+5 \mathrm{~min}$ at $\mathrm{pH} 6$ in citrate buffer solution for antigen retrieval. After boiling, the tissues that were kept to cool off in room heat for about 20 min were incubated for 5 min with hydrogen peroxide block solution to prevent endogenous peroxidase activity after being washed for $3 \times 5$ min with PBS (Phosphate Buffered Saline, P4417, Sigma-Aldrich, USA) -125-HP, Lab Vision Corporation, USA). In order to prevent dye remaining on the tissues that were washed with PBS for $3 \times 5$ min, Ultra $V$ Block (TA-125-UB, Lab Vision Corporation, USA) solution was applied for 5 min and they were incubated with $1 / 200$ diluted primary antibody (iris Rabbit Polyclonal H-067-17, Phoenix Pharmaceuticals, Inc., California, USA) at room temperature for $60 \mathrm{~min}$ in a humid environment. After the application of primary antibody, the tissues were incubated at room temperature for $30 \mathrm{~min}$ in a humid environment with a secondary antibody (biotinylated Goat Anti-Polyvalent (anti-mouse/ rabbit IgG), TP-125-BN, Lab Vision Corporation, USA) after being washed with PBS for $3 \times 5$ min. After the application of secondary antibody, the tissues were washed with PBS for $3 \times 5$ min and after being incubated at room temperature for 30 min in a humid environment with streptavidin peroxidase (TS-125-HR, LabVision Corporation, USA), were placed in PBS. After taking the image signal on a light microscope by dripping the solution of 3-amino-9-ethylcarbazole (AEC) Substrate + AEC Chromogen (AEC Substrate, TA-015 and HAS, AEC Chromogen, TA-002-HAC, LabVision Corporation, USA) on tissues, the tissues were washed with PBS synchronously. Tissues that underwent contrast dye with Mayer's haematoxylin were closed with appropriate closure solution (Large Volume Vision Mount, TA-125-UG, Lab Vision Corporation, USA) after being passed through PBS and distilled water. Prepared slides were evaluated and photographed on a Leica DM500 microscope (Leica DFC295).

The histopathological score was determined with respect to the prevalence $(0.1:<25 \%, 0.4$ : 26-50\%, 0.6: 51-75\%, 0.9: 76-100\%) and intensity $(0$ : absent, +0.5 : very low, +1 : low, +2 : moderate, +3 : severe) of immunological reactivity. Histopathological score was defined as prevalence times $(X)$ intensity.

\section{Statistical analysis}

Statistical analyses were performed using the SPSS 15.0 program. The normal distributions of TAS, TOS and immune variable groups were evaluated by the Kolmogorov-Smirnov test. One-way analysis of variance was used for group comparisons of TAS, TOS and immunity variables. Levene's statistic was used for the homogeneity test of variances. Tukey's pair-wise multiple comparison test was used to determine the differences between the groups of significant variables. The results were given as mean \pm SD. The level of significance was accepted to be at least $p<0.05$.

\section{Results}

\section{Body weight of rats}

In control (initial; $207.86 \pm 4.45 \mathrm{~g}$ ), final; 229.57 $\pm 5.19 \mathrm{~g}$ ) and benfotiamine (initial; $221.42 \pm 8.92 \mathrm{~g}$, final; $233.28 \pm 10.56 \mathrm{~g}$ ) groups, there was a statistically significant increase in body weights of rats at the end as compared to the beginning of the study $(p<0.05)$. The body weights of rats in MTX (initial; $212.0 \pm 4.47 \mathrm{~g}$, final; $214.29 \pm 12.79 \mathrm{~g}$ ) and MTX + benfotiamine groups (initial; $212.0 \pm 4.47 \mathrm{~g}$, final; $215.71 \pm 10.01 \mathrm{~g}$ ) increased at the end as compared to the beginning of the study. However, this increase was not statistically significant $(p>0.05)$ (Figure 1).

\section{TAS and TOS Levels}

In the biochemical study carried out to evaluate the serum TAS and TOS levels of all groups, TAS and TOS levels in trolox $\mathrm{Eq} / \mathrm{l}$ were similar in the control (TAS; $1.69 \pm 0.13$, TOS; $16.87 \pm 0.80$ ) and benfotiamine groups (TAS; $1.78 \pm 0.08$, TOS; $18.02 \pm 0.44)$. Compared with the control group, TAS levels $(1.36 \pm 0.09)$ in the MTX group significantly decreased while TOS levels $(20.58 \pm 1.58)$ significantly increased $(p<0.05)$. Compared with the MTX group, there was no significant difference

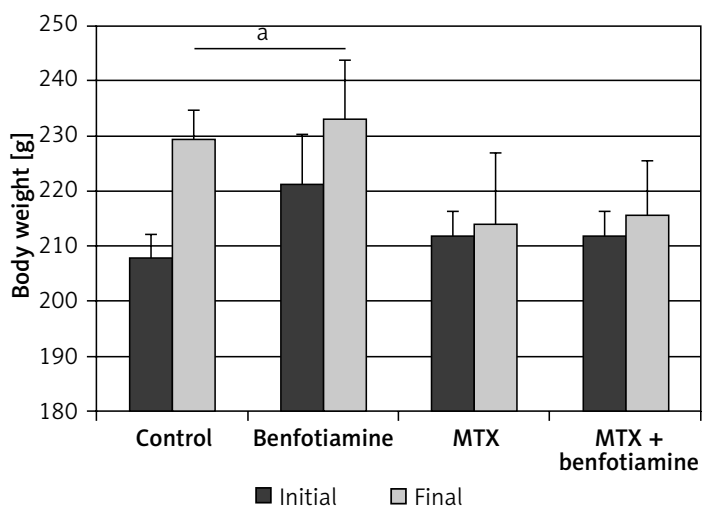

Figure 1. Initial and final body weights of rats are given as mean \pm standard deviation

${ }^{a}$ Compared with initial body weights $(p<0.05)$. 
A

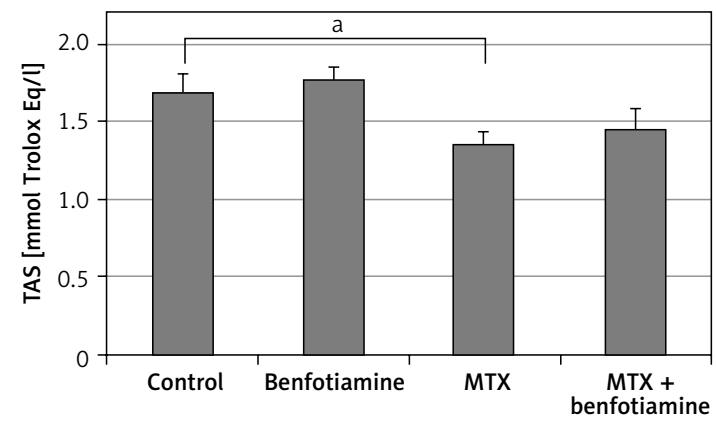

B

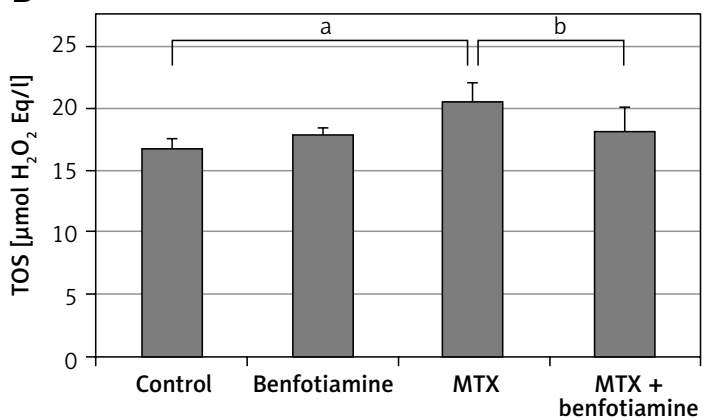

Figure 2. Serum TAS (A) and TOS (B) levels are given as mean \pm standard deviation

${ }^{a}$ Compared with control group, ${ }^{b}$ compared with MTX group $(p<0.05)$.

in TAS levels $(1.46 \pm 0.13)$ in the MTX + benfotiamine group, while TOS levels $(18.28 \pm 1.95)$ were significantly decreased $(p<0.05)$ (Figure 2 ).

\section{Histopathological results}

Examination with haematoxylin and eosin dye under the light microscope showed that the liver tissues of the control (Figure $3 \mathrm{~A}$ ) and benfotiamine (Figure $3 \mathrm{~B}$ ) groups were normal.

Compared with the control group, sinusoidal dilatation, oedema and haemorrhage (black star) and decreased glycogen (black arrow) in hepatocytes were clearly observed in the MTX group (Figure $3 \mathrm{C}$ ). However, in comparison with the MTX group, there was a significant decrease in sinusoidal dilatation, oedema and haemorrhage (black star), while decreased glycogen (black arrow) in hepatocytes persisted in the MTX + benfotiamine group (Figure $3 \mathrm{D}$ ).

\section{Immunohistochemical results}

As a result of examination of immunohistochemical staining for irisin immunoreactivity under the light microscope, irisin immunoreactivity was observed in hepatocytes (black arrow) in the liver tissue. Irisin immunoreactivity in the liver tissue was similar in control $(1.05 \pm 0.18)$ (Figure $4 \mathrm{~A}$ ) and benfotiamine $(1.14 \pm 0.33)$ (Figure $4 \mathrm{~B}$ ) groups. Compared with the control group, the irisin immu-
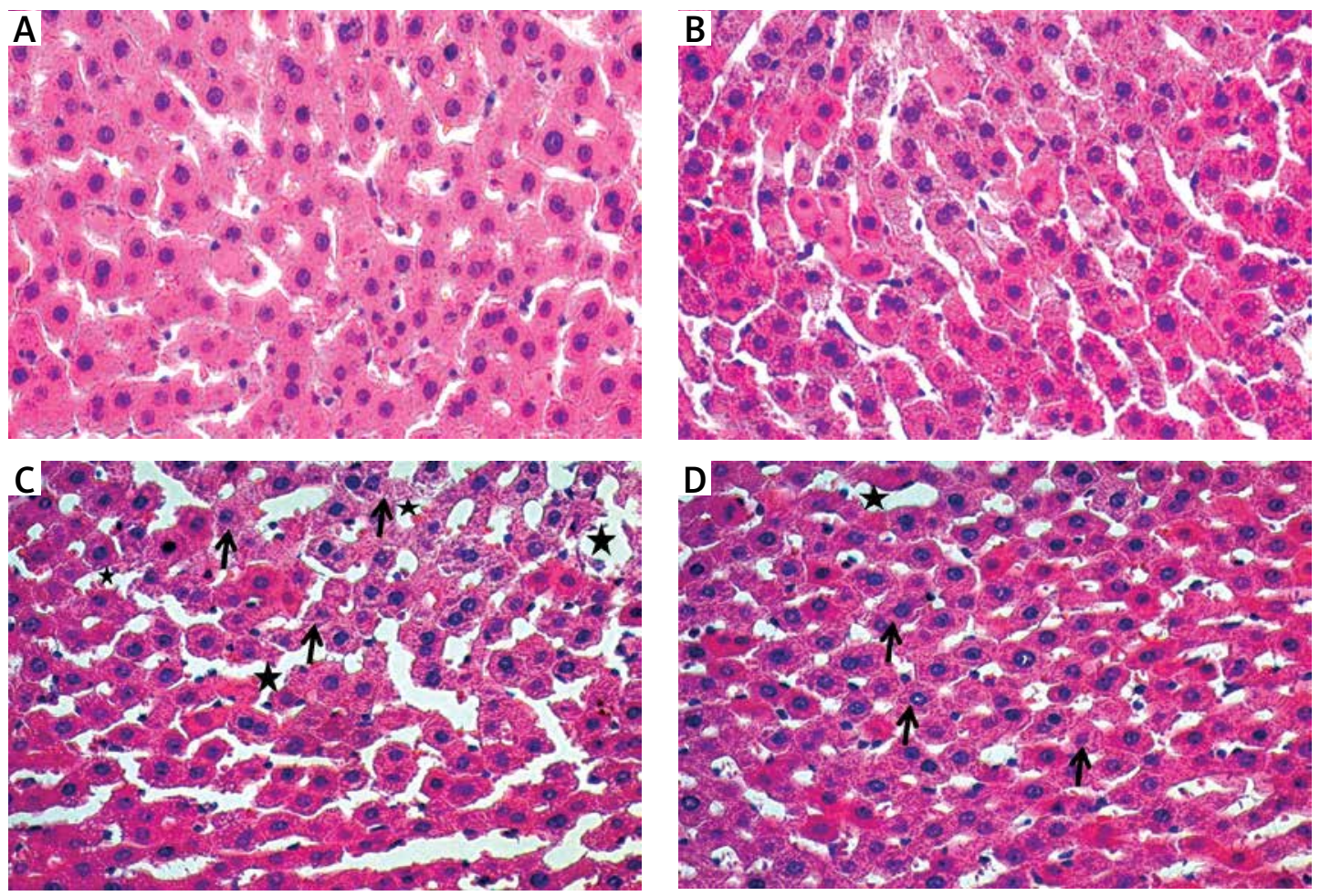

Figure 3. A - Normal looking liver tissue of control group. B - Normal looking liver tissue of benfotiamine group. C - Sinusoidal dilatation, oedema and haemorrhage (black star) in MTX group and decreased glycogen (black arrow) in hepatocytes. D - Significant decrease in sinusoidal dilatation, oedema and haemorrhage (black star) in MTX + benfotiamine group and decreased glycogen (black arrow) in hepatocytes 

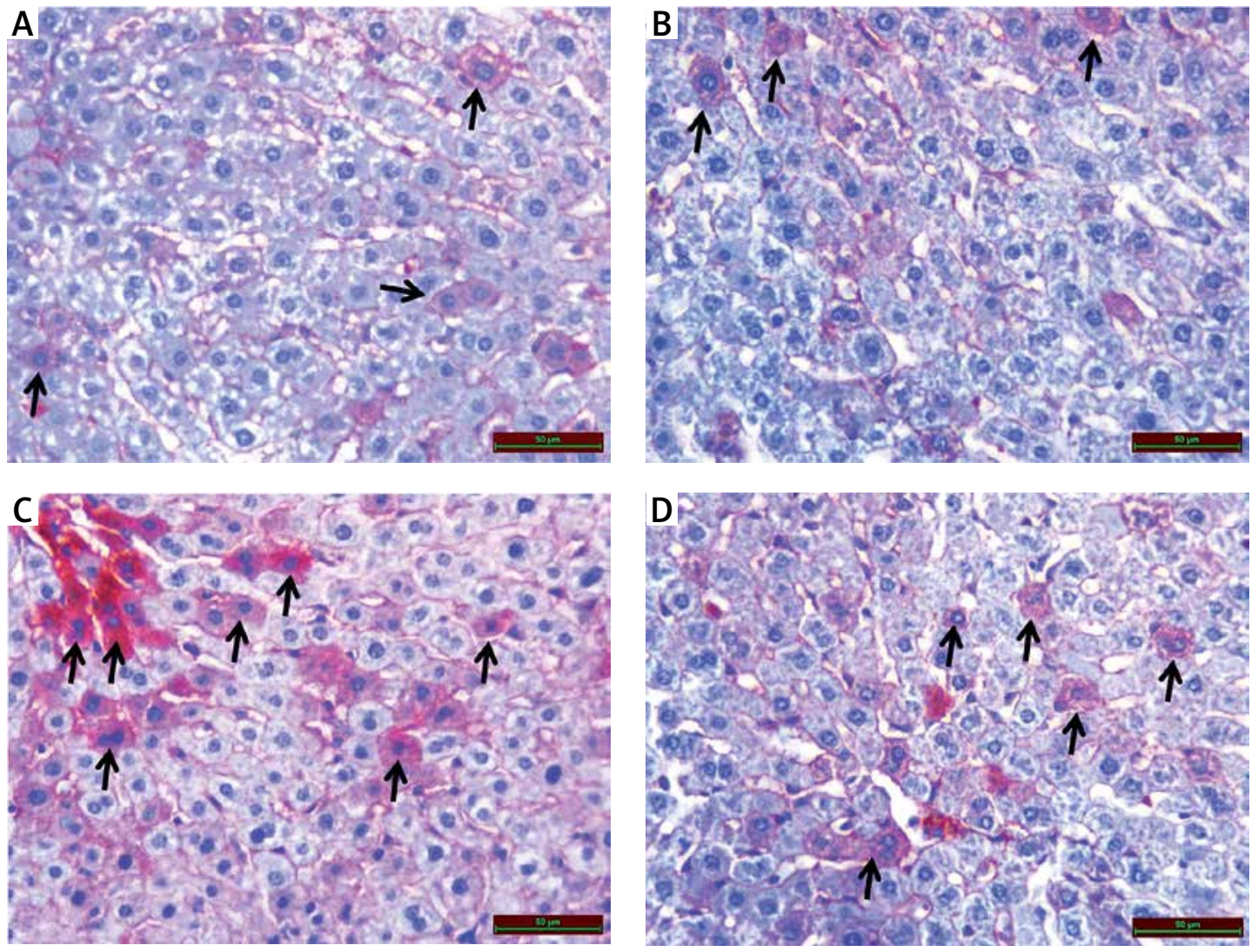

Figure 4. A - Irisin immunoreactivity (black arrow) in liver tissue of control group. B - Irisin immunoreactivity (black arrow) in liver tissue of benfotiamine group. C - Increased irisin immunoreactivity (black arrow) in liver tissue of MTX group. D - Irisin immunoreactivity (black arrow) in liver tissue of MTX + benfotiamine group

noreactivity in the MTX group $(1.90 \pm 0.67)$ was found to be statistically significantly increased $(p<0.05)$ (Figures $4 \mathrm{C}$ and 5$)$. Compared with the MTX group, the immunoreactivity of irisin was found to be decreased in the MTX + benfotiamine group (1.40 \pm 0.65$)$ (Figure $4 \mathrm{D})$, but this decrease was not statistically significant (Figure 5 ).

\section{Discussion}

In our study, we determined the oxidative stress in liver produced by MTX in rats according to increasing TOS and decreasing TAS levels. We observed increased irisin immunoreactivity in the damaged liver tissue. We found that benfotiamine, by decreasing oxidative stress caused by MTX, leads to histopathological improvement in liver and a decrease in irisin immune reactivity.

Methotrexate is a chemotherapeutic and immunosuppressive agent that can often be well tolerated. However, it causes hepatotoxicity, which can be life-threatening due to its cytotoxic effect. This situation has been shown in many experimental studies [20, 21]. Methotrexate has a toxic effect on the liver by many mechanisms. One of these mechanisms reduces the MTX folate level, suppresses DNA synthesis, while another mechanism causes it through oxidative

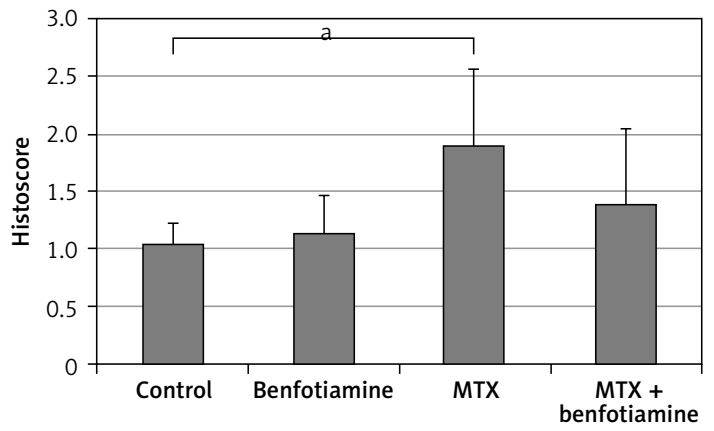

Figure 5. Histoscore of irisin immunoreactivity is given as mean \pm standard deviation

${ }^{a}$ Compared with control group $(p<0.05)$.

stress [22, 23]. In a study conducted by Bozkurt et al. on rats, it was observed that TAS levels were lowered after MTX and liver formed toxicity [24]. In another study conducted by Moghadam et al., a significant decrease was also observed in TAS levels in hepatotoxicity induced by MTX [25]. In our present study, MTX-induced oxidative stress in liver tissue of rats was detected by TOS increase and TAS decrease. Also, MTX led to severe liver damage. These results were compatible with the literature $[26,27]$. Our results suggest that MTX causes liver damage by increasing oxidative stress. 
Irisin suppresses inflammation, oxidative stress, thermogenesis, glucose uptake, gluconeogenesis and lipolysis, while increasing glycolysis, energy consumption and weight loss [28]. In a study conducted by Xiong et al., it was found that irisin increases energy consumption and causes hyperlipidaemia and hyperglycaemia [29]. Irisin is one of the factors responsible for the increase of energy consumption during possible oxidative stress. In oxidative stress, glycolysis is increased due to energy consumption. Irisin also meets this energy requirement by increasing glycolysis $[28,30]$. In previously conducted studies, it was found that irisin was an important antioxidant and anti-inflammatory agent [31-33]. In a study conducted by Rizk et al., it was shown that serum irisin levels increase against hepatotoxicity [34]. In our study, a significant increase in the immunoreactivity of irisin in the liver was observed in the MTX group. We also observed that MTX caused oxidative liver injury. In the MTX + benfotiamine group, oxidative stress and liver injury were clearly reduced. Although there is no statistically significant difference, the immunoreactivity of irisin in the liver was reduced in the MTX + benfotiamine group. All these results suggest that irisin plays a role in reduction of oxidative stress in the liver.

The antioxidant effect of benfotiamine has been shown in many studies with the help of superoxide dismutase, glutathione reductase and catalase [35]. Schmid et al., after experimentally inducing oxidative stresses in human, rat and pig kidneys by administering 3 different agents (mutagenic NQO, angiotensin II and uremic toxin indoxylsulphate), gave benfotiamine. It was observed that benfotiamine reduces the oxidative stress caused by these 3 agents [7]. Portari et al. found that in liver damage related to oxidative stress induced with ethanol, oxidative stress could be reduced with benfotiamine [36]. In our present study, a statistically significant decrease in TOS was observed with the addition of benfotiamine to oxidative stress caused by MTX; however, the increase in TAS level was statistically insignificant. This decrease in TOS and the increase in TAS have been linked to the antioxidant effects of benfotiamine. Benfotiamine significantly improved liver injury histopathologically. These results suggest that antioxidant properties of benfotiamine may contribute to prevention of liver damage caused by MTX.

In conclusion, in our study, we found that the immunoreactivity of irisin increases after liver toxicity induced by MTX. We observed that benfotiamine improved oxidative liver injury and reduced irisin immunoreactivity. We believe that irisin activity increases in order to reduce oxidative stress in liver toxicity and to meet the increased energy need. However, further experimental and clinical studies are required to confirm these findings indicating beneficial effects of irisin and benfotiamine before conducting clinical applications for treating liver injury in the light of biochemical and histopathological analysis.

\section{Conflict of interest}

The authors declare no conflict of interest.

\section{References}

1. Chabner BA, Wilson W, Supko J. Pharmacology and toxicity of antineoplastic drugs. Williams Hematology 2001; 8: 288-9.

2. Uraz S, Tahan V, Aygun C, et al. Role of ursodeoxycholic acid in prevention of methotrexate-induced liver toxicity. Dig Dis Sci 2008; 53: 1071-7.

3. Cetinkaya A, Bulbuloglu E, Kurutas EB, Kantarceken B. $\mathrm{N}$-acetylcysteine ameliorates methotrexate-induced oxidative liver damage in rats. Med Sci Monit 2006; 12: BR274-8.

4. Babiak RMV, Campello AP, Carnieri EG, Oliveira MBM Methotrexate: pentose cycle and oxidative stress. Cell Biochem Funct 1998; 16: 283-93.

5. Aydin S. Three new players in energy regulation. preptin, adropin and irisin. Peptides 2014; 56: 94-110.

6. Hofmann T, Elbelt U, Stengel A. Irisin as a muscle-derived hormone stimulating thermogenesis - a critical update. Peptides 2014; 54: 89-100.

7. Schmid U, Stopper H, Heidland A, Schupp N. Benfotiamine exhibits direct antioxidative capacity and prevents induction of DNA damage in vitro. Diabetes Metab Res Rev 2008; 24: 371-7.

8. Balakumar P, Rohilla A, Krishan P, Solairaj P, Thangathirupathi A. The multifaceted therapeutic potential of benfotiamine. Pharmacol Res 2010; 61: 482-8.

9. Gadau S, Emanueli C, Van Linthout S, et al. Benfotiamine accelerates the healing of ischaemic diabetic limbs in mice through protein kinase B/Akt-mediated potentiation of angiogenesis and inhibition of apoptosis. Diabetologia 2006; 49: 405-20.

10. Bozic I, Savic D, Stevanovic I, Pekovic S, Nedeljkovic N, Lavrnja I. Benfotiamine upregulates antioxidative system in activated BV-2 microglia cells. Front Cell Neurosci 2015; 9: 351.

11. Turan M, Cayir A, Cetin N, Suleyman H, Turan IS, Tan H. An investigation of the effect of thiamine pyrophosphate on cisplatin-induced oxidative stress and DNA damage in rat brain tissue compared with thiamine: thiamine and thiamine pyrophosphate effects on cisplatin neurotoxicity. Hum Exp Toxicol 2014; 33: 14-21.

12. Turan MI, Cetin N, Turan IS, Ozgeris FB, Suleyman H. Effects of thiamine and thiamine pyrophosphate on oxidative stress by methotrexate in the rat brain. Lat Am J Pharm 2013; 32: 203-7.

13. Cichoż-Lach H, Michalak A. Oxidative stress as a crucial factor in liver diseases. World J Gastroenterol 2014; 20: 8082-91.

14. Halliwell B. Antioxidant characterization: methodology and mechanism. Biochem Pharmacol 1995; 49: 1341-8.

15. Aydogdu A, Karakas EY, Erkus E, et al. Epicardial fat thickness and oxidative stress parameters in patients with subclinical hypothyroidism. Arch Med Sci 2017; 13: 383-9. 
16. Majer M, Gackowski D, Różalski R, Siomek-Górecka A, Oliński R, Budzyński J. Systemic oxidoreductive balance and vascular function in individuals without clinical manifestation of atherosclerosis. Arch Med Sci Atheroscler Dis 2017; 2: e37-45.

17. Trocha M, Merwid-Ląd A, Pieśniewska M, et al. Age-related differences in function and structure of rat livers subjected to ischemia/reperfusion. Arch Med Sci 2018; 14: 388-95.

18. Erel O. A novel automated direct measurement method for total antioxidant capacity using a new generation, more stable ABTS radical cation. Clin Biochem 2004; 37: 277-85.

19. Erel O. A new automated colorimetric method for measuring total oxidant status. Clin Biochem 2005; 38: 1103-11.

20. Hafez HM, Ibrahim MA, Ibrahim SA, Amin EF, Goma W, Abdelrahman AM. Potential protective effect of etanercept and aminoguanidine in methotrexate-induced hepatotoxicity and nephrotoxicity in rats. Euro J Pharmacol 2015; 768: 1-12.

21. Akbulut S, Elbe H, Eris C, et al. Cytoprotective effects of amifostine, ascorbic acid and $\mathrm{N}$-acetylcysteine against methotrexate-induced hepatotoxicity in rats. World J Gastroenterol 2014; 20: 10158.

22. Jolivet J, Cowan KH, Curt GA, Clendeninn NJ, Chabner BA. The pharmacology and clinical use of methotrexate. N Engl J Med 1983; 309: 1094-104.

23. Jahovic N, Çevik H, Şehirli AÖ, Yeğen BÇ, Şener G. Melatonin prevents methotrexate-induced hepatorenal oxidative injury in rats. J Pineal Res 2003; 34: 282-7.

24. Bozkurt M, Bodakci M, Turkcu G, et al. Protective effects of carvacrol against methotrexate-induced liver toxicity in rats. Acta Chir Belg 2014; 114: 404-9.

25. Moghadam AR, Tutunchi S, Namvaran-Abbas-Abad A, et al. Pre-administration of turmeric prevents methotrexate-induced liver toxicity and oxidative stress. BMC Complement Altern Med 2015; 15: 246.

26. Bozkurt M, Em S, Oktayoglu P, et al. Carvacrol prevents methotrexate-induced renal oxidative injury and renal damage in rats. Clin Invest Med 2014; 37: 19-25.

27. Daggulli $M$, Dede O, Utangac MM, et al Protective effects of carvacrol against methotrexate-induced testicular toxicity in rats. Int J Clin Exp Med 2014; 7: 5511-6.

28. Mahgoub MO, D'Souza C, AlDarmaki RS, Baniyas MM, Adeghate $\mathrm{E}$. An update on the role of irisin in the regulation of endocrine and metabolic functions. Peptides 2018; 104: 15-23.

29. Xiong XQ, Chen D, Sun HJ, et al. FNDC5 overexpression and irisin ameliorate glucose/lipid metabolic derangements and enhance lipolysis in obesity. Biochim Biophys Acta 2015; 1852: 1867-75.

30. Balogh E, Veale DJ, McGarry T, et al. Oxidative stress impairs energy metabolism in primary cells and synovial tissue of patients with rheumatoid arthritis. Arthritis Res Ther 2018; 20: 95.

31. Mazur-Bialy A, Kozlowska K, Pochec E, Bilski J, Brzozowski T. Myokine irisin-induced protection against oxidative stress in vitro. Involvement of heme oxygenase-1 and antioxidazing enzymes superoxide dismutase-2 and glutathione peroxidase. J Physiol Pharmacol 2018; 69: 117-25.

32. Zhu D, Wang H, Zhang J, et al. Irisin improves endothelial function in type 2 diabetes through reducing oxidative/ nitrative stresses. J Mol Cell Cardiol 2015; 87: 138-47.

33. Bosma M, Gerling M, Pasto J, et al. FNDC4 acts as an anti-inflammatory factor on macrophages and improves colitis in mice. Nat Commun 2016; 7: 11314.
34. Rizk FH, Elshweikh SA, Abd El-Naby AY. Irisin levels in relation to metabolic and liver functions in Egyptian patients with metabolic syndrome. Can J Physiol Pharmacol 2015; 94: 359-62.

35. Harisa Gl. Benfotiamine enhances antioxidant defenses and protects against cisplatin-induced DNA damage in nephrotoxic rats. J Biochem Mol Toxicol 2013; 27: 398-405.

36. Portari GV, Ovidio PP, Deminice R, Jordão Jr AA. Protective effect of treatment with thiamine or benfotiamine on liver oxidative damage in rat model of acute ethanol intoxication. Life Sci 2016; 162: 21-4. 